\title{
MEASUREMENT OF THE INTENSITY OF THE BEAM IN THE ABORT GAP AT THE TEVATRON UTILIZING SYNCHROTRON LIGHT*
}

\author{
R. Thurman-Keup ${ }^{\#}$, E. Lorman, T. Meyer, S. Pordes, FNAL, Batavia, IL 60510, U.S.A. \\ S. De Santis, LBNL, Berkeley, CA 94720, U.S.A.
}

\begin{abstract}
This paper discusses the implementation of abort gap beam intensity monitoring at the Tevatron collider at Fermilab. There are two somewhat independent monitors which measure the intensity of the synchrotron light emitted by particles in the abort gaps. One system uses a gated Photomultiplier Tube (PMT) to measure the light intensity, and the other system uses a single lens telescope, gated image intensifier, and Charge Injection Device (CID) camera to image the beam.
\end{abstract}

\section{MOTIVATION}

During operation of the Tevatron in colliding beam mode, particles diffuse out of bunches and are no longer captured in RF buckets. Unlike an electron storage ring where free particles are rapidly swept out of the ring from energy losses due to synchrotron radiation, free particles in a proton storage ring continue to circulate for several minutes before succumbing to the much smaller radiative losses. This means that as particles diffuse out of the bunch they drift around the ring forming a DC component to the beam.

The presence of DC beam in the abort gaps (see Fig. 1) has serious implications. During an abort, the kicker magnets ramp up during the abort gap. Beam passing through the kickers while they are ramping sprays into superconducting magnets causing quenches, and into the silicon detectors of the collider experiments where the silicon suffers severe radiation damage. To combat this problem, the Tevatron has an electron lens (TEL) which is turned on during the abort gap to eject particles from the gap. However, until now, the only measure of the amount of beam left in the abort gap was the count rate in a set of scintillation counters surrounding the beamline that were gated in time with the abort gap. These measurements suffer from the fact that the counters are measuring the beam leaving the abort gap, not the beam still in it. This document describes two partially independent methods for directly measuring the beam in the abort gap using synchrotron light: gated photomultiplier tube, and intensified CID camera.

\section{THEORY}

When a charged particle traverses a magnetic field, it experiences a transverse acceleration and emits synchrotron radiation. Under certain conditions, sufficient radiation is produced in the visible part of the

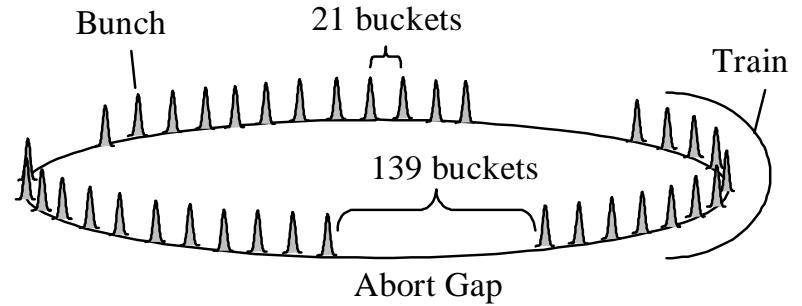

1113 RF buckets total RF bucket $=18.8 \mathrm{~ns}$

Figure 1: Beam structure of Tevatron. There are 36 bunches arranged in 3 trains of 12 separated from each other by abort gaps.

spectrum such that measurements can be made using photocathode type detectors.

The Tevatron proton beam consists of 36 bunches with each bunch typically containing $250 \times 10^{9}$ protons. Table 1 lists typical values for beam intensities and expected synchrotron radiation signals assuming a DC beam intensity of 1 part in $10^{4}$ of the total beam intensity. As one can see, the optical intensity of a bunch is several thousand times larger than that from the abort gap. This large difference produces a requirement that the detector must be able to see the small amount of light immediately after a bunch while not being blinded by the bunch itself.

Table 1: Typical beam intensities and optical intensities associated with Tevatron operation for both the gated PMT and the CID camera system. When referring to DC beam or abort gaps, the assumption is that there are $10^{9}$ particles in the DC beam component.

\begin{tabular}{|l|c|c|}
\hline \multirow{2}{*}{ Quantity } & \multicolumn{2}{|c|}{ Typical Value } \\
\cline { 2 - 3 } & PMT & CID \\
\hline Total Beam Intensity & $10^{13}$ particles \\
Desired DC Beam Sensitivity & $10^{9}$ particles \\
\hline \# of $\gamma$ / turn (25 nm bw @ 400 nm) & \multicolumn{2}{|c|}{800,000} \\
/ bunch (250 x 10 protons) & \multicolumn{2}{|c|}{3} \\
/ rf bucket of DC beam & \multicolumn{2}{|c|}{420} \\
\hline / abort gap & $100 \mathrm{~nm}$ & $10 \mathrm{~nm}$ \\
\hline Wavelength Acceptance & $35 \%$ & $20 \%$ \\
\hline Optical Efficiency & \multicolumn{2}{|c|}{$18 \%$} \\
\hline Photocathode Efficiency & \multicolumn{2}{|c|}{} \\
\hline \# of photoelectrons / turn & 200,000 & 10,000 \\
/ bunch (250 x 10 ${ }^{9}$ ) & 0.8 & 0.04 \\
/ rf bucket of DC beam & 106 & 6 \\
/ abort gap & \multicolumn{2}{|c|}{} \\
\hline
\end{tabular}

\footnotetext{
* Funded by Universities Research Association Inc. under Contract No. DE-AC02-76CH03000 with the U.S. Department of Energy.

\# keup@fnal.gov
} 


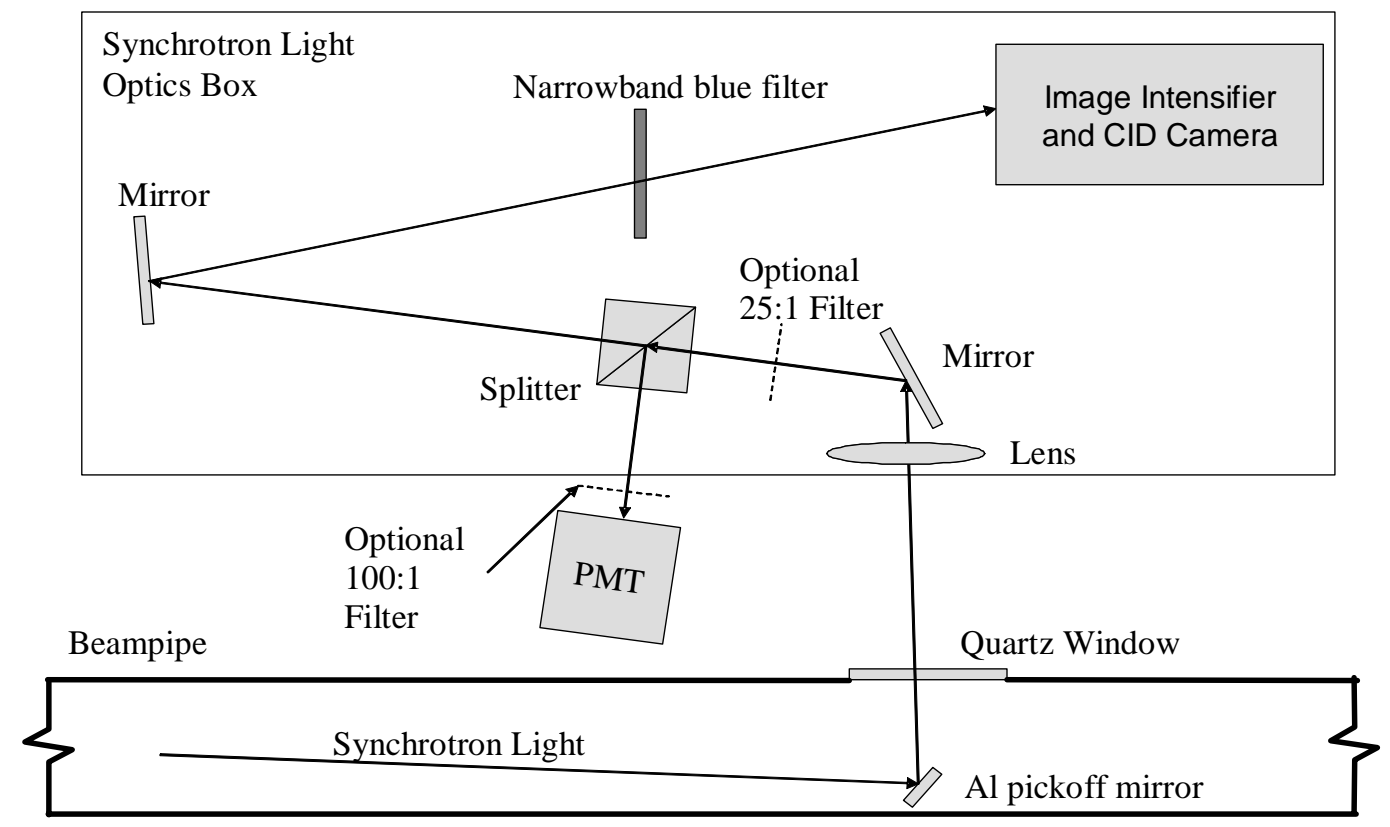

Figure 2: Diagram of synchrotron light apparatus. There is a lens in the system to focus the light for use in beam profile measurements by the CID camera system.

\section{APPARATUS}

The facility for intercepting and detecting the synchrotron light consists of a moveable mirror mounted inside the beampipe, a quartz vacuum window, and a light-tight box containing the detectors (see Fig. 2).

There are two detectors in the optics box: a gated PMT and a gated image intensifier connected to a CID camera. The image intensifier / CID camera is used mainly for beam profile measurements, while the photomultiplier tube is dedicated to the abort gap beam intensity measurement. They share the same synchrotron light beam via an optical beam splitter. The gated PMT has the advantage of much larger gain and larger bandwidth producing a faster measurement of the beam intensity.

\section{Gated PMT}

Two versions of the gated PMT have been used. One version consists of a side-window tube with 2 of the dynodes connected to a pulsing circuit. In the off state, the dynodes are held at the wrong voltage effectively shutting off the multiplication chain. In the on state, the dynode voltages are moved to their proper levels and the PMT functions normally. This system works well with two exceptions. The behavior of the tube immediately after being gated on exhibits transients related to the amount of light present just before the gate. Fortunately, the transients are small for typical bunch intensities at the Tevatron. The second problem is capacitive coupling of the gating pulse to the anode output, making it unusable for $150 \mathrm{~ns}$ after the gate starts.

The version of the gated PMT presently installed in the Tevatron is a Hamamatsu R5916U-50 micro-channel plate (MCP) PMT with a minimum gating time of 5 ns on loan to FNAL from LBNL. This tube does not have either of the problems the other PMT version had. Hence this tube can be used to measure the DC beam immediately following a bunch. It does however have less gain $\left(\leq 10^{6}\right.$ compared to $10^{7}$ ) and a maximum duty cycle of only $1 \%$.

The data acquisition (DAQ) system consists of a fast integrator, to which the anode of the PMT is connected, and a VME digitizer which is read by an application on a processor board residing in the VME crate. Data is collected for 1000 revolutions of the beam and averaged in the processor board. This cycle is repeated every 3 or 4 seconds. The application controls the timing of both the PMT and integration gates.

\section{Image Intensifier / CID Camera}

The image intensifier system is normally used for beam bunch profile measurements, and as such the gain of the system is not large. However, if enough camera frames are summed together, a measurement of the DC beam can be made. The DAQ system consists of a framegrabber card which captures the RS-170 video signal coming from the CID camera, and a Labview program running on a generic Windows PC. Images of the beam are integrated to produce an intensity measurement.

\section{CALIBRATION}

Both systems do background subtractions and apply a scale factor. There are two methods that can be used to obtain the scale factor. The first involves comparing bunch intensity measurements by these systems to those from the dedicated bunch intensity instrumentation. For the gated PMT, this is accomplished by inserting the optical attenuators, positioning the gates over a bunch, and measuring the intensity. The CID system routinely 
measures the intensity of bunches as part of the beam profile measurement.

The second method of obtaining the scale factor makes use of the electron lens (TEL) to change the amount of DC beam. Figure 3 shows the result of turning off the TEL. When the TEL is turned off, particles that would have been ejected by the TEL are now allowed to accumulate around the ring and the loss rate goes down. In the plot, this is manifested by a decrease in the rate of change of the total beam intensity as measured by a resistive wall current monitor. The change in the total beam intensity from TEL on to TEL off is the extra DC beam that has been allowed to accumulate, and the ratio formed between it and the abort gap systems is the scale factor. The advantage of this method is that the scale factor is obtained without inserting filters or moving gates.
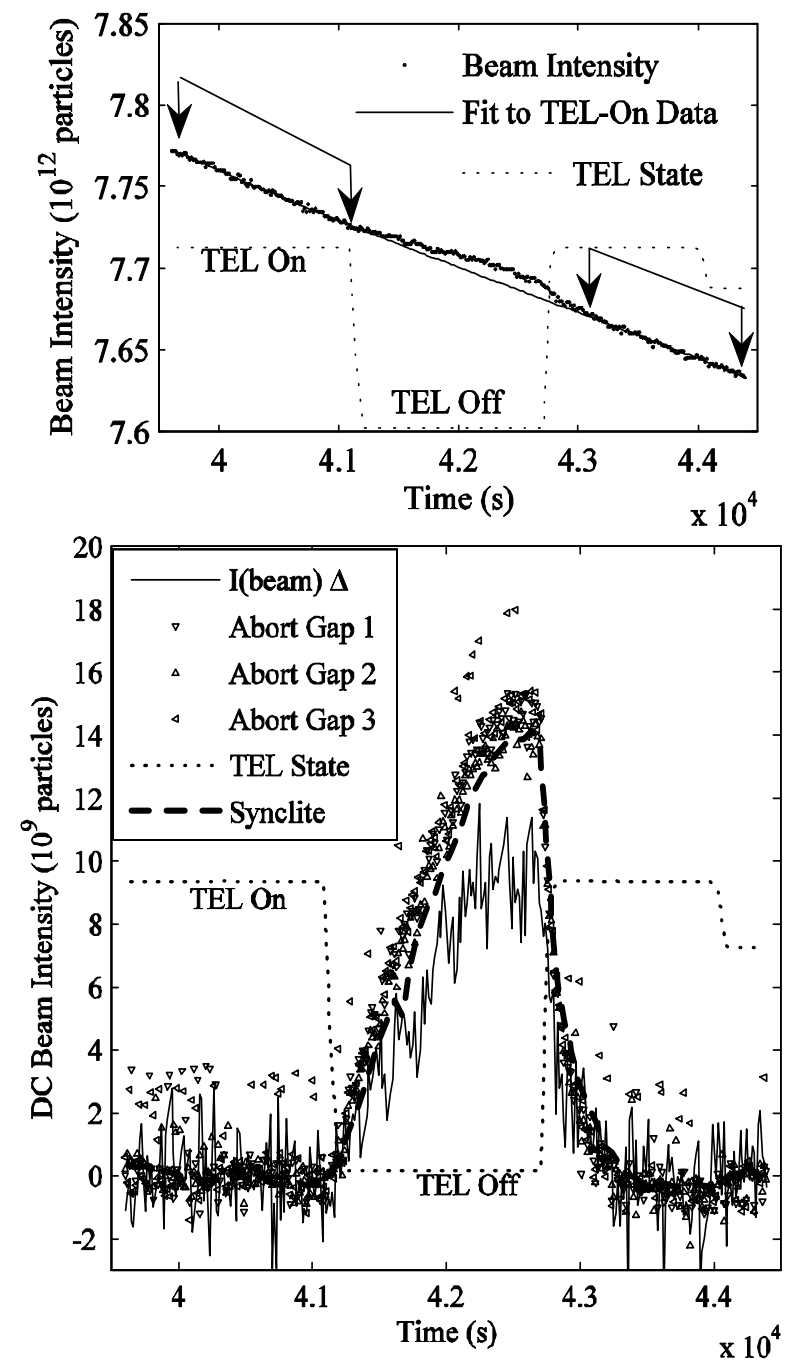

Figure 3: TEL-on / TEL-off calibration. Top) Behavior of total beam intensity as the TEL is turned off and back on. The arrows indicate the regions that are fit with a quadratic to obtain the TEL-on baseline. Bottom) The baseline-subtracted total beam intensity, labeled I(beam) $\Delta$ in the plot, is compared to baseline-subtracted measurements from the PMT in the 3 abort gaps and to the CID camera system (a.k.a. Synclite).

\section{SUMMARY}

Figure 4 shows typical abort gap beam levels at the beginning of a collider store and indicates some features that are present. Monitoring the abort gap during an entire store points to the initial abort gap beam being left over captured remnants from the injection process, whereas the TEL eliminates beam that has diffused out of bunches before it can be recaptured.

The current PMT system has been in use for nearly a year and is incorporated into the daily operations of the Tevatron and monitored by the collider experiments. The abort gap intensity must be below some safe value before the control program will end the store. There have been several instances of longitudinal damper problems where the abort gap beam intensity measurement grew very large reflecting the fact that beam was being shaken from bunches and deposited around the ring. This information enabled experts to identify and rectify the situation.

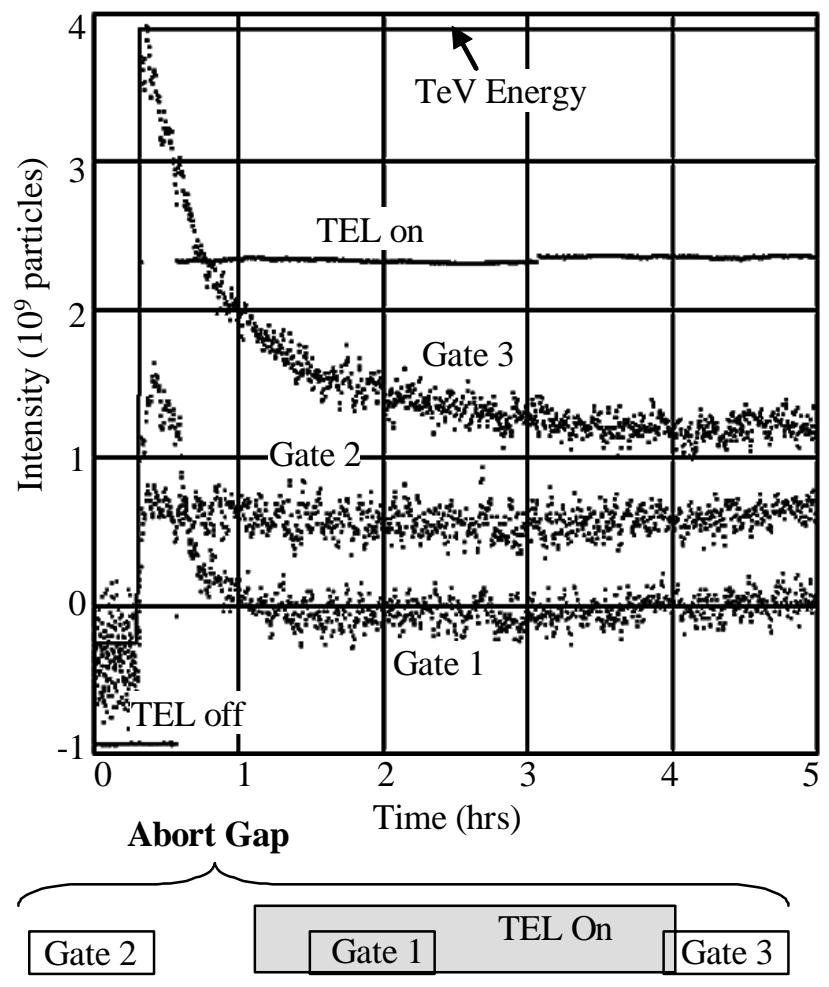

Figure 4: Typical abort gap beam intensity behavior at the beginning of a store. No signal is present until the beam energy is at its operating level of $980 \mathrm{GeV}$. The TEL turns on $\sim 15$ minutes later. At that time, gate 1, which overlaps the TEL, is cleaned out. The beam that is in the abort gap when the energy reaches $980 \mathrm{GeV}$ is a captured remnant left over from coalescing. The bottom diagram shows the relative timing of the gates and the TEL.

\section{ACKNOWLEDGEMENTS}

We would like to thank A. Hahn, H. Cheung, J. Fast, K. Schultz, M. Ruschman, C. Lindenmeyer, R. Miksa, S. Hansen, H. Schneider, M. Binkley, S. Valishev, D. Miller, C. Lundberg, V. Shiltsev and R. Dixon for various contributions to this project. 